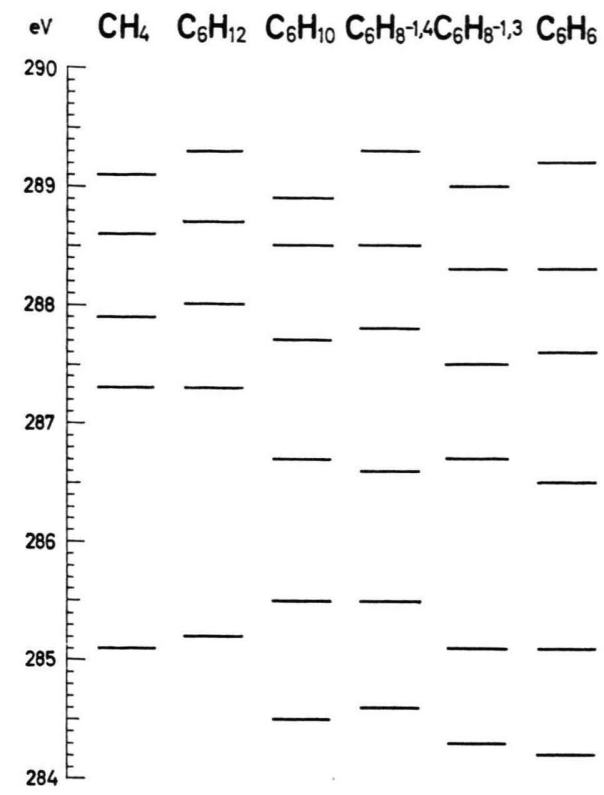

Abb. l. Termschema der Feinstruktur der $\mathrm{C}_{\mathrm{K}} \cdot$ Kante von Methan, Cyclohexan, Cyclohexen, Cyclohexadien-1,4, Cyclohexadien-1,3 und Benzol.

eine gruppentheoretische Diskussion der Auswahlregeln näher begründeten, dem Übergang eines 1s-Elektrons in unbesetzte, nichtbindende $2 \pi^{*}$-Molekülorbitale, die aus der Linearkombination (LCAO) von $2 \mathrm{p}_{z}$-Atomfunktionen des Kohlenstoffs entstehen.

Der Vergleich der Spektren von Cyclohexadien-1,4 und -1,3 zeigt, daß das gesamte Termschema gegenüber den Verbindungen mit isolierten Doppelbindungen zu kleineren Energien verschoben ist, wenn konjugierte Doppelbindungen im Molekül vorhanden sind. Ver-

5 T $\mathrm{AE}-$ KJu $\mathrm{H}_{\mathrm{A}}$, Dissertation, Frankfurt (Main) 1963. gleicht man die Termschemata aller untersuchten ungesättigten Verbindungen mit denen von Methan und Cyclohexan, so fällt auf, daß der Unterschied zwischen denen der gesättigten und denen der ungesättigten Verbindungen deutlicher als der zwischen den Termfolgen bei letzteren ist. Das Spektrum von Cyclohexen zeigt, daß offenbar eine einzige Doppelbindung ausreicht, um das gesamte Spektrum in Richtung der Spektren von Molekülen mit mehreren Doppelbindungen zu verschieben.

Die Schwierigkeiten der Aufnahmetechnik der Spektren und ihrer Auswertung erlaubten es bisher in den meisten Fällen noch nicht, zuverlässige Werte für die Lagen der Absorptionskanten, d. h. der 1s-Ionisierungsenergien zu bekommen. Die 1s-Ionisierungsenergie von $\mathrm{CH}_{4}$ erhielten wir aus der beobachteten Termfolge unter Zugrundelegung einer wasserstoffähnlichen Serienformel zu 288,9 eV. Der früher nach der gleichen Methode ermittelte Wert ${ }^{1}$ beträgt $289,04 \mathrm{eV}$, während ihn $\mathrm{HA}_{\mathrm{A}}{ }^{5}$ mit Hilfe eines Pseudoneonmodells für $\mathrm{CH}_{4} \mathrm{zu}$ $286 \mathrm{eV}$ theoretisch berechnete. Ein Vergleich der 1sIonisierungsenergien verschiedener Verbindungen würde Aussagen über die mögliche Beeinflussung der Energien der 1s-Elektronen durch die Valenzelektronen liefern und zu einer Überprüfung des Modells des „starren Rumpfes" bei Molekülberechnungen beitragen können. Ab-initio-Rechnungen von Preuss und Diercksen ${ }^{6}$ im Rahmen einer SCF-MO-LC(LCGO)-Methode zeigen jedenfalls, daß die „1s-Einteilchenenergien“ von Benzol, Cyclopentadienylanion, Cyclopropan, Äthylen, C, $\mathrm{C}^{2^{+}}$sowie $\mathrm{C}^{4^{+}}$verschieden sind und daß daher die inneren Elektronen von den Bindungsvorgängen wesentlich beeinflußt sein müssen.

Der Fritz ter M e e r - Stiftung dankt der eine von uns (G.) für die finanzielle Unterstützung während dieser Untersuchung.

${ }^{6}$ H. Preuss u. G. Diercksen, Arbeitsberichte der Gruppe Quantenchemie, Max-Planck-Institut für Physik und Astrophysik No. 2, 82 [1966].

\section{Epitaktische Silberaufdampfschichten mit Wolframunterkeimung}

\section{Klaus Müller * und Gerhard SchimpF}

Institut für Theoretische Physik der Technischen Hochschule Karlsruhe

(Z. Naturforschg. 22 a, 1814-1816 [1967] ; eingeg. am 11. September 1967)

Beim Aufdampfen epitaktisch wachsender Ag-Einkristallschichten auf frischgespaltener Glimmerunterlage (mit Unterlagentemperatur $250{ }^{\circ} \mathrm{C}$ ) ist nach ${ }^{1}$ ein An-

* Z. Zt.: Bell Telephone Laboratories, Inc. Murray Hill, N.J., USA.

1 K. Müller, Z. Phys. 195, 105 [1966].

2 D. W. Pashley u. M. J. Stowell, Proc. $5^{\text {th }}$ Intern. Congr. Electron Microscopy, Philadelphia 1962. fangsintervall zu beobachten, während dessen sich nur Inseln bilden, der elektrische Schichtwiderstand also praktisch unendlich bleibt. Erst danach wachsen die Inseln zusammen, der Widerstand fällt annähernd exponentiell ab. Er bleibt aber nach Beendigung des Aufdampfens bei sehr dünnen Schichten (Größenordnung $10^{2} \AA$ ) gewöhnlich nicht konstant, sondern nimmt wegen ihres flüssigkeitsähnlichen Verhaltens ${ }^{2-4}$ unter regellosen Sprüngen wieder zu.

Einkristallische Ag-Schichten herzustellen, deren Widerstand praktisch vom Beginn des Bedampfens an regelmäßig abfällt und nach Beendigung der Schicht-

3 K. J. Hanszen, Z. Naturforschg. 19 a, 820 [1964].

4 E. F. Pócza, A. Barna u. P. Barna, Proc. Intern. Symposium, held at Clausthal-Göttingen, 1965, p. 153. 

K. Müller und G. Schimpf, Epitaktische Silberaufdampfschichten mit Wolframunterkeimung (S. 1814).

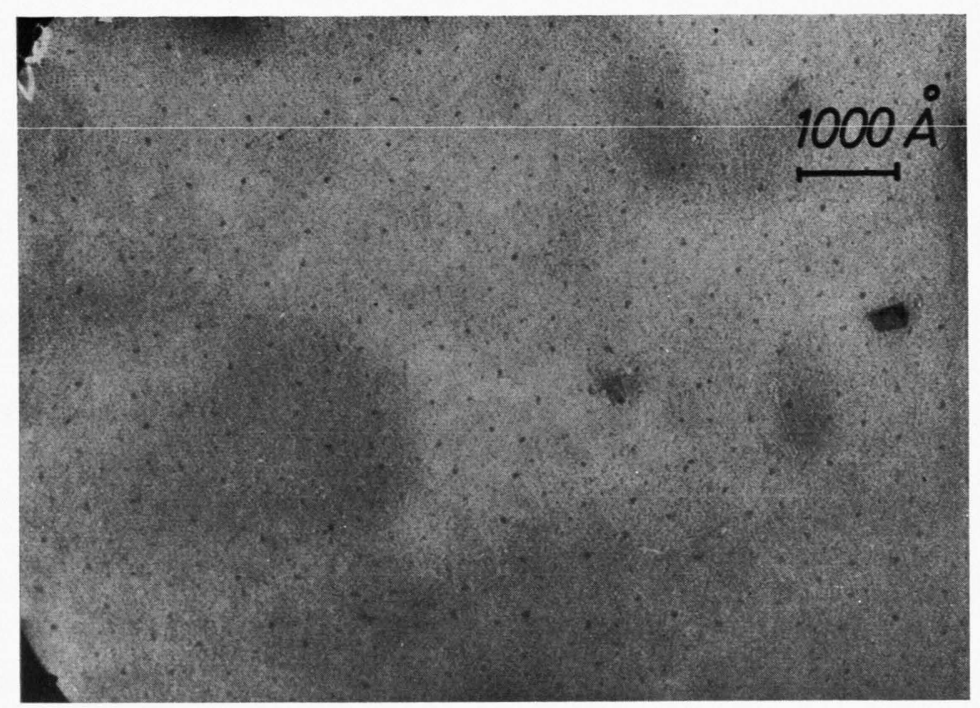

Abb. 1. Wolframkeime auf Glimmer. Vergrößerung $100000 \times$; $1 \mathrm{~mm} \triangleq 100 \AA$. 
herstellung seinen Endwert beibehält, ist uns dadurch gelungen, $\mathrm{da} ß$ wir die frischgespaltene Glimmerunterlage vor der Bedampfung mit Silber mit dem viel höher schmelzenden und daher wenig beweglichen Wolfram schwach vorbekeimten ${ }^{5}$. Die an Luft hergestellte Glimmerspaltfläche wurde hierzu zunächst - für die Widerstandsmessungen - rasch mit Elektroden aus Leitgold versehen und dann in einem Hochvakuum-Hilfsrezipienten bei Zimmertemperatur mittels eines zuvor entgasten, glühenden Wolframdrahtes mit der Aufdampfrate von etwa $0,3 \AA / \min 2$ Minuten lang mit $\mathrm{W}$ beschossen. Danach gelangte der Schichtträger in den Ultrahochvakuum. Rezipienten. Vom Spalten des Glimmers bis zum Auspumpen der UHV-Apparatur vergingen etwa 20 Minuten. Nach 20 -stündigem Ausheizen bei $450{ }^{\circ} \mathrm{C}$ wurden als Druck einige $10^{-10}$ Torr erreicht. Beim Ausglühen des Verdampfungsofens und nachfolgendem Verdampfungsvorgang zur Erzeugung der einkristallischen AgSchicht (Ofen $1100^{\circ} \mathrm{C}$, Glimmerunterlage $250^{\circ} \mathrm{C}$, Aufdampfraten des $\mathrm{Ag}$ in Einzelfällen zwischen 20 und $50 \AA / \mathrm{min}$, Längenangaben sind an ToLANskys optisches Verfahren angeschlossen) stieg er auf einige $10^{-8}$ Torr, um aber sofort danach wieder auf einige $10^{-9}$ Torr zurückzugehen. Der elektrische Widerstand der Silberschichten mit solcher Wolframunterkeimung verhält sich tatsächlich weitgehend normal: Die Anlaufzeit mit unendlichem Schichtwiderstand wird sehr klein. Der Widerstand fällt danach exponentiell mit der Zeit ab und verharrt, sobald man den Dampfstrahl abstellt - auch schon bei Dicken unter $100 \AA-$, stets völlig konstant auf dem erreichten Wert. Offenbar entstehen jetzt an den vielen W-Keimen kleine Ag-Inseln, die sich rasch zu einer leitenden Schicht zusammenschließen und auch nachher keine größeren Bewegungen mehr ausführen. Mit Beugung langsamer Elektronen geprüft, zeigt auch eine so unterkeimte Silberschicht das Beugungsbild der (111) -Ebene als Oberfläche. Das epitaktische Wachstum auf Glimmer zum Einkristall wird durch die vergleichsweise geringe Menge von Wolframkeimen offenbar nicht merklich gestört.

In welchem Bereich die Keimzahldichte des W liegen darf, um zu brauchbaren Ergebnissen zu führen, ist uns noch unbekannt. Wir haben aber die mit Erfolg benutzte Unterkeimung quantitativ untersucht. Sie entsteht, wie oben beschrieben, durch Aufdampfen von Wolfram mit einer mittleren Schichtdicke von $0,6 \AA$. Das Ergebnis zeigt die elektronenmikroskopische Aufnahme Abb. $1^{*}$. Die W-Atome sind zu größeren Keimen zusammengerutscht, die regellos über die Glimmerunterlage zerstreut liegen. Nach elektronenmikroskopischen Stereoaufnahmen lassen sie sich in Annäherung beschreiben als verhältnismäßig hohe Säulen, deren Grundflächendurchmesser im Mittel $40 \AA$ und deren Höhe rund $30 \AA$ beträgt. Ihre Anzahldichte ergibt sich durch Auszählen solcher Bilder zu 10,6 $10^{10} / \mathrm{cm}^{2}$. Berechnet man hieraus das Gesamtvolumen der Säulen und damit eine mittlere Schichtdicke für gleichmäßig gedachte Verteilung des $\mathbf{W}$, so erhält man gute Über-

5 Diese Methode wurde von Herrn Dr. J. J. Lander angeregt. * Abb. 1 auf Tafel S. 1814 b. einstimmung mit dem oben aus Zeit und Aufdampfrate erhaltenen Wert.

Interessant ist auch ein Vergleich der Keim- bzw. Inseldichten des Silbers im Anfangsstadium des Wachs. tums unter verschiedenen Bedingungen. Man kann sie sich für den Fall nicht bekeimter Unterlage dadurch verschaffen, daß man das Volumen von lauter halbkugelförmig und gleichmäßig verteilt gedachten Inseln in dem Augenblick mit dem gesamten bis dahin aufgedampften Silbervolumen gleichsetzt, in dem die Inseln zur leitenden Schicht zusammenfließen. Zeit und Aufdampfrate sind dazu aus dem jeweiligen Aufdampfversuch, die Zeit insbesondere durch den scharfen Einsatz des Widerstandsabfalls bekannt. Im Fall der vorbekeimten Glimmerunterlage darf angenommen werden, daß die Zahl der anfänglichen Silberinseln mit derjenigen der W-Keime übereinstimmt, an die sie sich ja anlagern. So ergeben sich die Werte der Tab. 1 .

\begin{tabular}{lcccc}
\hline & $\begin{array}{c}\text { Unter- } \\
\text { lagen- } \\
\text { tempe- } \\
\text { ratur } \\
\text { in }{ }^{\circ} \mathrm{C}\end{array}$ & $\begin{array}{c}\text { mittl. Schichtdicke } \\
\text { bei Zusammenschluß } \\
\text { in } \AA\end{array}$ & $\begin{array}{c}\text { Ag-Insel- mittlerer } \\
\text { dichte } \\
\text { in } \mathrm{cm}^{-2}\end{array}$ & $\begin{array}{c}\text { Insel- } \\
\text { abstand } \\
\text { in } \AA\end{array}$ \\
\hline $\begin{array}{l}\text { unbekeimt } \\
\text { unbekeimt } \\
\text { bekeimt }\end{array}$ & 250 & 71,5 & $\begin{array}{c}13,4 \cdot 10^{10} \\
1,0 \cdot 10^{10}\end{array}$ & 273 \\
mit & 250 & 258 & & \\
$0,6 \AA \mathrm{W}$ & 250 & $<50$ & $10,6 \cdot 10^{10}$ & 308 \\
\hline
\end{tabular}

Tab. 1.

Unsere Ergebnisse ohne Unterkeimung schließen sich gut an solche an, die Снорга ${ }^{6}$ durch Auszählen elektronenmikroskopischer Aufnahmen erhielt, wobei die Bedampfung bei variierter mittlerer Schichtdicke abgebrochen war (Abb. 2).

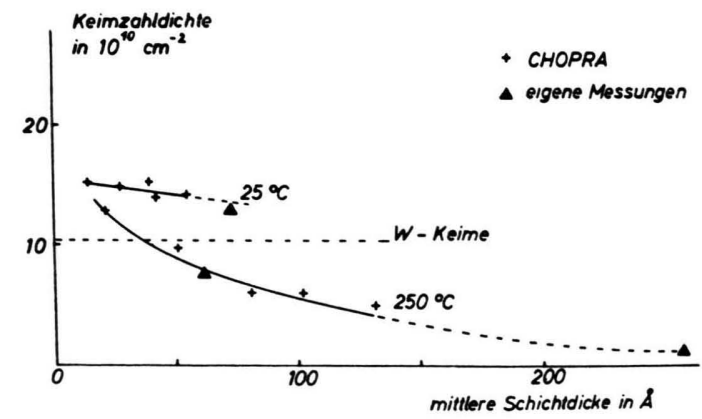

Abb. 2. Keimzahldichte für Silber auf reinem Glimmer.

Man sieht zunächst, wie sehr ohne Vorbekeimung die Inselzahl mit wachsender Temperatur zurückgeht und dadurch die Gleichmäßigkeit des Ag-Schichtaufbaus verschlechtert. Man sieht ferner, daß demgegenüber mit W-Keimen die Zahl der Silberinseln bei hohen Temperaturen (Epitaxietemperatur!) verzehnfacht werden

${ }^{6}$ K. L. Chopra, J. Appl. Phys. 37, 3405 [1966]. 
kann, so daß - wie ohne W-Keime bei Zimmertemperatur - die Inseln sich rasch zur leitenden Schicht zusammenschließen und die Schicht gleichmäßig und zeitlich stabil werden kann. Die Epitaxietemperatur liefert zudem die einkristallische Struktur.
Herrn Prof. Dr. Franz Wolf, der die Arbeit ermöglichte, danken wir für ständige Förderung aller Art, Herrn Dr. L. Albert für elektronenmikroskopische Aufnahmen und der Deutschen Forschungsgemeinschaft für die Bereitstellung von Mitteln.

\section{Nachweis von Antimon-125 in Hüttenblei}

\section{W. KolB}

Physikalisch-Technische Bundesanstalt Braunschweig und Berlin

(Z. Naturforschg. 22 a, 1816-1818 [1967] ; eingeg. am 29. September 1967)

From scintillation spectrometer measurements it results that certain kinds of recently produced lead may be contaminated with ${ }^{125} \mathrm{Sb}$ probably from atmospheric fallout. The activity concentration of ${ }^{125} \mathrm{Sb}$ in a crude lead sample made from ore concentrate of very low radioactivity was found to be $4 \mathrm{fCi} / \mathrm{g}$. This low value is sufficient to produce additional photopeaks in the background spectrum of a low level scintillation counter, if the material is used as the detector shielding. The ${ }^{125} \mathrm{Sb}$ concentration in lead containing a few percent of antimony will be even higher. For refined lead, however, the probability of contamination is rather low because ${ }^{125} \mathrm{Sb}$ is separated during antimony raffination in the lead smelter.

Für die Abschirmung von Meßeinrichtungen zur Bestimmung sehr geringer Aktivitäten bzw. Aktivitätskonzentrationen werden Materialien besonders geringer Eigenaktivität benötigt, und zwar nach Möglichkeit solche mit hoher Ordnungszahl, wie z. B. Blei. Im allgemeinen kann das natürliche Blei jedoch das radioaktive Isotop ${ }^{210} \mathrm{~Pb}^{1-5}$ mit seinen Folgeprodukten enthalten, von denen sich ${ }^{210} \mathrm{~m} B$ wegen seiner harten $\beta$ Strahlung besonders störend auswirkt; deshalb hat man für solche Zwecke auf altes Blei zurückgegriffen, dessen ${ }^{210} \mathrm{~Pb}-\mathrm{Gehalt}$ inzwischen abgeklungen war. In einer früheren Arbeit ${ }^{6}$ wurde gezeigt, daß es daneben auch möglich ist, durch Auswahl geeigneter Erzsorten Blei sehr geringer Eigenaktivität herzustellen, besonders wenn durch die Art des Verhüttungsverfahrens Zuschlagstoffe (z. B. Bleischrott, Koks) vermieden werden können, die einen nicht unbeträchtlichen Beitrag zur Eigenaktivität liefern. Letzteres ist als der Hauptgrund dafür anzusehen, daß schwedisches ElektroofenBlei zu den aktivitätsärmsten handelsüblichen Bleisorten zählt.

Für die Szintillationsspektrometrie kommt es nicht allein auf einen niedrigen integralen Nulleffekt an, sondern auch darauf, daß das Nulleffektspektrum möglichst aus einem Kontinuum ohne Linien besteht. Andernfalls wird die quantitative Bestimmung einzelner Nuklide nach der Abzugsmethode erschwert, wenn sich deren Linien mit den Linien im Nulleffekt überlagern. Eine größere Meßunsicherheit ist dann die Folge.

${ }^{1}$ H. L. de Vries u. G. W. Barendsex, Physica 19, 987 [1953].

$2 \mathrm{G}$. W. B ARENDSEN, Ouderdomsbespaling met radioactive kool stof, Diss. Groningen 1955.

3 H. I. Weller, H. C. Anderson u. J. L. Barker Jr., Nature 206, 1211 [1965].
In Ergänzung zu der oben erwähnten Arbeit ${ }^{6}$ wurden deshalb verschiedene Bleisorten im Szintillationsspektrometer untersucht, um festzustellen, ob und gegebenenfalls welche Linien im Spektrum von radioaktiven Verunreinigungen der inneren Bleiabschirmung herrühren. Als Detektor diente ein 4 " $\varnothing \times 4^{\prime \prime}-\mathrm{NaJ}(\mathrm{Tl})$ Bohrloch-Szintillationszähler, der außen mit $10 \mathrm{~cm}$ handelsüblichem und innen mit $2 \mathrm{~cm}$ altem Blei abgeschirmt war. Im Inneren dieser Bleiabschirmung befand sich die $\mathrm{zu}$ untersuchende Bleisorte in Form eines unmittelbar den Kristall umgebenden Zylinders, dessen Wanddicke etwa $4 \mathrm{~mm}$ betrug und der mit einer Kreisscheibe gleicher Dicke oben abgedeckt war.

Für die Untersuchung wurden drei Bleisorten ausgewählt, die sich durch niedrige Eigenaktivität auszeichnen. Bei der Probe „Palca“ handelt es sich um eine in einem Treibofen der Friedrich-August-Hütte Nordenham nach dem Tarnowitz-Verfahren mit Koksgrus ohne weitere Zuschlagstoffe hergestellte Sonderschmelze aus peruanischem Erzkonzentrat ${ }^{6}$. Bei der mit „Boliden" gekennzeichneten Probe handelt es sich um handelsübliches Hüttenweichblei (Charge Nr. B 1) der Bolidens Gruvaktie-Bolag in Skelleftehamn (Schweden), das im Elektroofen gewonnen wurde. Die Altbleiprobe stammt aus der Bergung eines vor mehr als 100 Jahren gesunkenen Segelschiffs. In der Tab. 1 sind zunächst die im Szintillationsspektrometer gemessenen Gesamtimpulsraten für den Energiebereich 60 bis $960 \mathrm{keV}$ angegeben. Zum Vergleich enthält die Tabelle außerdem die in einem Antikoinzidenz- $\beta$-Zähler gemessenen $\beta$-Impulsraten sowie die radiochemisch bestimmte ${ }^{210} \mathrm{~Pb}-\mathrm{Kon}$ zentration.

\begin{tabular}{lccc}
\hline Probe & $\begin{array}{c}\gamma \text {-Impulsrate } \\
60 \mathrm{bis} \mathrm{960 \textrm {keV }} \\
\mathrm{min}^{-1}\end{array}$ & $\begin{array}{c}\beta \text {-Impulsrate } \\
\mathrm{min}^{-1} / \mathrm{dm}^{2}\end{array}$ & $\begin{array}{c}{ }^{\mathbf{2 1 0} \mathrm{Pb}-} \\
\text { Konzentration } \\
\mathrm{pCi} / \mathrm{g}\end{array}$ \\
\hline Palca & 133 & 4,5 & 0,9 \\
Boliden & 123 & 1,9 & 0,4 \\
Altblei & 120 & 0,5 & - \\
\hline
\end{tabular}

Tab. 1.

Während die Spektren, die mit den Proben „Boliden" und "Altblei" aufgenommen wurden, sich nur durch den unterschiedlichen ${ }^{210} \mathrm{~Pb}-\mathrm{Gehalt}$ und die dadurch bedingte ${ }^{210 \mathrm{~m}} \mathrm{Bi}-\mathrm{Bremsstrahlung}$ unterschieden, wurden im Spektrum der Probe „Palca“ Linien fest-

4 B. Grinberg u. Y. Le Gallic, Int. J. appl. Radiat. Isotopes 12, 104 [1961].

5 O. Paakkola u. H.-J. Steinert, Atompraxis 9, 232 [1963].

6 W. A. Kolв, Die Eigenaktivität von Blei. in: Proceedings of the First International Congress of IRPA, Pergamon Press, Oxford, im Druck. 